\title{
Editorial
}

\section{Indian PM's International Yoga Day 2016 Advocacy: Trends, Advances and Future Perspectives}

\author{
Akshay Anand ${ }^{1}$, Shirley Telles ${ }^{2}$ \\ ${ }^{1}$ Neuroscience Research Laboratory, Department of \\ Neurology, PGIMER, Chandigarh, ²Patanjali Research \\ Foundation, Haridwar, India
}

The decision of Prime Minister Sh. Narendra Modi to role model and advocate Yoga as national health initiative on 2nd International Yoga Day in Chandigarh inspired the local administration to engage citizens and experts in regular yoga training and interdisciplinary dialogue. A series of yoga training programs ensued in 180 city locations and a proposal for analysis of its effects in blood/vitals of these individuals was mooted. Launch of 3D awareness campaigns in the Lake premises and organizing a 4 day national conference 'Yoga for Good life' were the other highlights.

An interdisciplinary conference (June 9-12, 2016) was held in collaboration with Ministry of AYUSH, Mumbai University, Panjab University and Annals of Neurosciences, coordinated by Ms Kirti Garg, Prof Shubadra Joshi and Col G S Chaddha. This dwelled on Yoga's role in Health, Medicine, Profession, Personality, Education and Management. The speakers included Honorable Swami Ramdev, Honorable Ravi Shankar, and Honorable Acharya Balkrishna and was attended by over 300 delegates. Many important policy coordination meetings in the sidelines were coordinated by $\mathrm{Dr}$ Jaideep Arya. The proceedings of the conclave are being published in the current issue. Mr Ajay Balaji Joshi, the nodal officer for PM's visit cum Deputy commissioner of Chandigarh spelled out the recommendations of the conclave. These included: inclusion of Sanskrit, Philosophy and Ayurveda in all educational courses in Universities/Medical Institutes as ancient Ayurveda texts, containing knowledge of Indian traditional medicine are in Sanskrit; it was argued that Yoga is irrelevant without its prescription and research in Medical institutes. It was felt that the Depts of Complementary Medicine/Ageing could be established by Medical Institutes so that patients visiting these OPDs have the freedom to choose Ayurved/Yoga intervention under common facility, making healthcare affordable for complex diseases. This will improve quality of medical care and generate comparative data between Yoga and drugs. For best integration, MCI could include representatives from alternative medicine, scientists, patient advocacy groups and quality assurance representatives. A suggestion for initiating the PhD-MD course in Integrative medicine also came up. The Advisor, Sh Parimal Rai, also reinforced to establish a hospital for traditional medicine in Chandigarh.

The Govt of India had proposed the AYUSH IYD protocol of Yoga to be implemented for the International Yoga Day public engagement under the chairmanship of Honourable HR Nagendra. Its effects on vital parameters in a month long yoga practice could be interesting. The Chandigarh administration with support of Bharat Vikas Parishad Charitable Foundation, Joshi Foundation and DGHS, Dr Vanita Gupta and her team plan to study such effects. The retrospective analysis of Chandigarh based healthy yoga trainers, who were engaged in such a huge exercise, could provide interesting data from their health profiles, if analysed.

When healthy naïve Yoga trainees were introduced to Yoga as run up for the International Yoga Day, some of them volunteered to undertake General Health Questionnaire, before PM's Chandigarh's visit on June 21. The analysis of the health profiles of several of these naive yoga practitioners could generate new data and opportunity for scientists to undertake future studies based on large public exercises like this. Majority of the public practised AYUSH mandated Yoga protocol between 6-6.45 am. The biggest display was at Panjab University grounds where participants came in huge numbers and appeared euphoric after 1 month of yoga practice. Many variables can be analysed after more time to see if this can protect them from complex diseases, if they continue the practice of yoga. The trainers reported that yoga volunteers felt relaxed, slept well and could concentrate better after 1 month of yoga and wanted to continue Yoga for a longer time. Many of them are still continuing to do Yoga in Chandigarh parks which can still be seen flooded with neo Yoga practitioners. The preliminary observations reveal that there was a general trend towards increased health consciousness in Chandigarh due to PM's advocacy. This brought people closer to Yoga. These observations were based on compliance to daily attendance, of both trainers and trainees, which was monitored by the organisers to ensure PM's public Yoga engagement. Long term controlled studies are needed to examine if yoga's preventive impact on degenerative diseases lasts until old age. 
Integrative

Medicine

\begin{tabular}{l|l}
\hline Integr Med Int 2016;3:113-114 \\
\hline DOI 10.1159/000452262 & $\begin{array}{l}\text { @ 2016 The Author(s) Published by S. Karger AG, Basel } \\
\text { www.karger.com/imi }\end{array}$ \\
\hline
\end{tabular}

Indian PM's International Yoga Day 2016 Advocacy: Trends, Advances and Future Perspectives

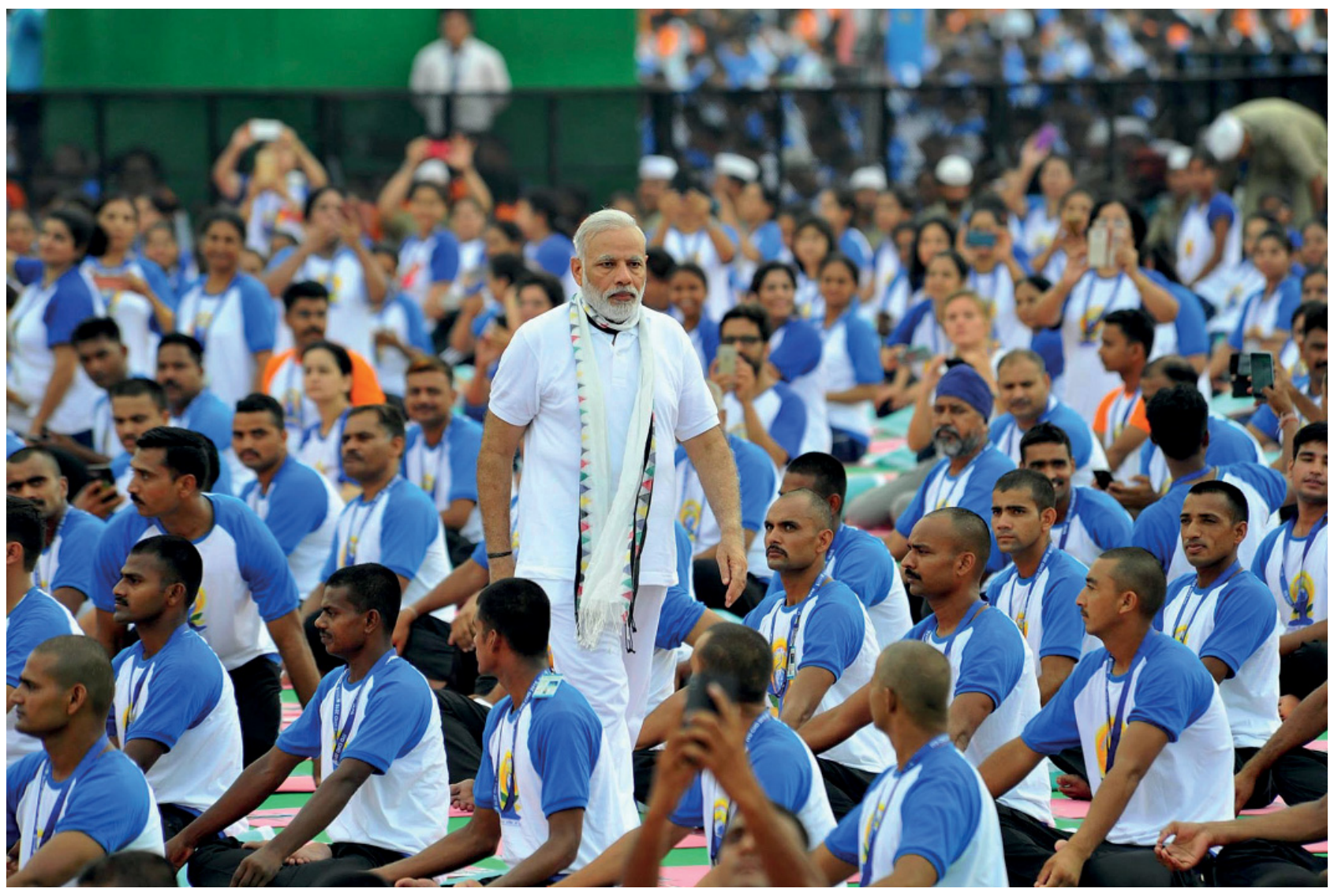

Fig. 1. Prime Minister Sh. Narendra Modi on 2nd International Yoga Day in Chandigarh. 